\title{
Article
}

\section{SSEA-4 Antigen Is Expressed on Rabbit Lymphocyte Subsets}

\author{
Jaromír Vašíček ${ }^{1,2, * \mathbb{D}}$, Andrej Baláži ${ }^{1}$, Miroslav Bauer ${ }^{1,3}$ (1) and Peter Chrenek ${ }^{1,2, *}$ \\ 1 NPPC, Research Institute for Animal Production Nitra, Institute of Farm Animal Genetics and Reproduction, \\ Hlohovecká 2, 95141 Lužianky, Slovakia; andrej.balazi@nppc.sk (A.B.); miroslav.bauer@nppc.sk (M.B.) \\ 2 Department of Biochemistry and Biotechnology, Faculty of Biotechnology and Food Science, \\ Slovak University of Agriculture in Nitra, Tr. A. Hlinku 2, 94976 Nitra, Slovakia \\ 3 Department of Botany and Genetics, Faculty of Natural Sciences, Constantine the Philosopher University in \\ Nitra, Nábrežie Mládeže 91, 94974 Nitra, Slovakia \\ * Correspondence: jaromir.vasicek@nppc.sk (J.V.); peter.chrenek@uniag.sk (P.C.); \\ Tel.: +421-37-654-6600 (J.V.); +421-37-641-4274 (P.C.)
}

Citation: Vašíček, J.; Baláži, A.; Bauer, M.; Chrenek, P. SSEA-4 Antigen Is Expressed on Rabbit Lymphocyte Subsets. Magnetochemistry 2021, 7, 94. https://doi.org/10.3390/

magnetochemistry7070094

Academic Editor: Marie Frenea-Robin

Received: 2 March 2021

Accepted: 24 June 2021

Published: 27 June 2021

Publisher's Note: MDPI stays neutral with regard to jurisdictional claims in published maps and institutional affiliations.

\section{Copyright: (c) 2021 by the authors.} Licensee MDPI, Basel, Switzerland. This article is an open access article distributed under the terms and conditions of the Creative Commons Attribution (CC BY) license (https:/ / creativecommons.org/licenses/by/ $4.0 /)$.

\begin{abstract}
SSEA-4 antigen can be mainly found in embryos and embryonic stem cells. However, its expression has been observed also in adult stem and progenitor cells, or even in some differentiated cells. Moreover, we found a considerable number of SSEA-4 positive (SSEA- ${ }^{+}$) cells within the rabbit peripheral blood and bone marrow mononuclear cells (PBMCs and BMMCs) in our previous study. Since no information about such cells can be found anywhere in the literature, the aim of this study was to identify their origin. At first, phenotypic analyses of fresh rabbit PBMCs and BMMCs were performed using flow cytometry and specific antibodies against SSEA-4 and leukocyte subsets. Then, SSEA $-4^{+}$were enriched using magnetic activated cell sorting (MACS) and analyzed for their phenotype using qPCR. We found significant SSEA $-4^{+}$cell population in PBMCs $(\sim 50 \%)$ and BMMCs $(\sim 20 \%)$. All those cells co-expressed CD45 and a majority of them also expressed B-cell marker (IgM; $50 \%$ of SSEA $-4^{+}$PBMCs and $60 \%$ of SSEA $-4^{+}$BMMCs). Increased $(p<0.05)$ expression of SSEA-4, CD45 and B-cell markers (IgM, CD79 $\alpha$ and MHCII) were also noticed by qPCR in SSEA-4 ${ }^{+}$cells enriched via MACS (with efficiency over $80 \%$ ). Both methods did not detect significant expression of monocyte or T-cell markers. In conclusion, SSEA $-4^{+}$cells in rabbit blood and bone marrow are of hematopoietic origin and probably belong to B-lineage cells as possessing the phenotype of $\mathrm{B}$ lymphocytes. However, the true function of SSEA-4 antigen in these cells should be explored by further studies.
\end{abstract}

Keywords: SSEA-4; rabbit; peripheral blood; bone marrow; flow cytometry; MACS; qPCR

\section{Introduction}

Stage-specific embryonic antigen-4 (SSEA-4) is an epitope on related globoseries glycosphingolipids [1], which is widely used as a cell surface marker to identify human pluripotent stem cells. SSEA-4 together with SSEA-3 are abundantly expressed in preimplantation human embryos and human embryonic stem cells or embryonal carcinoma cells, where they are downregulated after cell differentiation [1-4]. In addition, SSEA-4 was previously described also in somatic stem cells as dental pulp stem cells, or umbilical cord blood-derived very small embryonic-like stem cells [5], and also in bone marrow-derived mesenchymal stem cells (MSCs) [6]. Moreover, SSEA-4 has been recently found in progenitor cells such as cardiomyocyte progenitors or pancreatic ductal progenitor cells $[7,8]$. However, the SSEA-4 function and its role as a pluripotent marker is largely unknown and remains controversial [9-12].

Although the SSEA-4 expression is strictly regulated on human embryonic stem cells and during the preimplantation development itself, this expression is not essential for the pluripotency regulation [12]. It was demonstrated that the depletion of glycosphingolipids (including SSEA4) had no effect on the undifferentiated state. On the other hand, it is crucial for the differentiation of cells or survival of already differentiated cell lineages 
during development [13]. Nevertheless, SSEA-4 expression was also observed on the cell surface of some highly differentiated cells such as neurons [14], erythrocytes [1] or corneal and limbal epithelial cells [15]. Furthermore, SSEA-4 positive cells were found in adult marmoset testes [16]. Thus, SSEA-4 seems to be a universal carbohydrate antigen that should not be strictly used only for the evaluation and/or isolation of stem cells.

As mentioned above, expression of SSEA- 4 antigen has been noticed also in the MSCs of different species, such as mouse and human [6], goat and monkey [17] as well as rabbit $[18,19]$. Moreover, neural progenitor cells [20] or MSCs [6] had been isolated based on the SSEA-4 expression using fluorescence- (FACS) or magnetic-activated cell sorting (MACS), respectively. The latter mentioned method was used in our previous study focused on the direct isolation of SSEA $-4^{+}$cells from rabbit bone marrow in order to facilitate the establishment of MSCs [21]. However, that study failed to achieve this goal since such a method was not more effective than the classical plastic adherent method. In any case, this study revealed a finding that rabbit bone marrow can comprise about $25 \%$ of SSEA-4 positive (SSEA- ${ }^{+}$) cells that are evidently not only of MSCs origin. Furthermore, our preliminary unpublished experiments indicate that SSEA $-4^{+}$cell can be found also in rabbit peripheral blood.

Therefore, the aim of the presented study was to identify the phenotype of SSEA $-4^{+}$ cells presented in the rabbit peripheral blood and bone marrow by the combination of flow cytometry, magnetic sorting and quantitative Real-Time PCR analyses.

\section{Results}

\subsection{Flow-Cytometric Phenotyping of SSEA-4 $4^{+}$Cells}

Freshly isolated mononuclear cells from rabbit peripheral blood (PBMCs) and bone marrow (BMMCs) were stained with SSEA- 4 antibody in order to identify SSEA $-4^{+}$cells. Those cells can be mainly found within the lymphocyte gate as demonstrated by the forward (FSC-H) and side scatter (SSC-H) plots (Figures 1A and 2A). Co-staining with the antibodies against CD45, CD14, IgM, panT2, CD4 and CD8 was used to identify the cell type of SSEA- $4^{+}$cells (Figures $1 \mathrm{~B}$ and $2 \mathrm{~B}$ ). More than $50 \%$ of PBMCs were positive for SSEA-4 marker. In addition, all of them co-expressed CD45, leukocyte common antigen, and almost one-half of those cells was positive for B-cell marker (IgM). On the other hand, SSEA $-4^{+}$cells were nearly negative for CD14 (monocytes), and panT2, CD4 and CD8 (T-cells; Figure 1B).

In the case of BMMCs, only about $20 \%$ of cells were positive for SSEA- 4 marker. Similarly, the majority of them co-expressed also CD45 and about $60 \%$ were positive for IgM. Again, the cells were negative for CD14 and all T-cell markers (panT2, CD4 and CD8; Figure 2B).

\subsection{Magnetic-Activated Cell Sorting (MACS) of SSEA-4 $4^{+}$Cells}

According to the flow cytometry, MACS technique achieved a high purity and efficiency as the positive fractions contained more than $90 \%$ of SSEA-4 positive PBMCs and about $85 \%$ of SSEA-4 positive BMMCs (Table 1). Moreover, cells in both positive fractions, PBMCs and BMMCs, co-expressed also CD45 (90\% and 80\%, respectively). The same percentage was observed for the bound magnetic beads marked by LCR, thus confirming the high MACS efficiency. On the other hand, negative fractions contained a significantly lower number of SSEA-4 ${ }^{+}$cells in comparison to control (unsorted) samples (Table 1). 

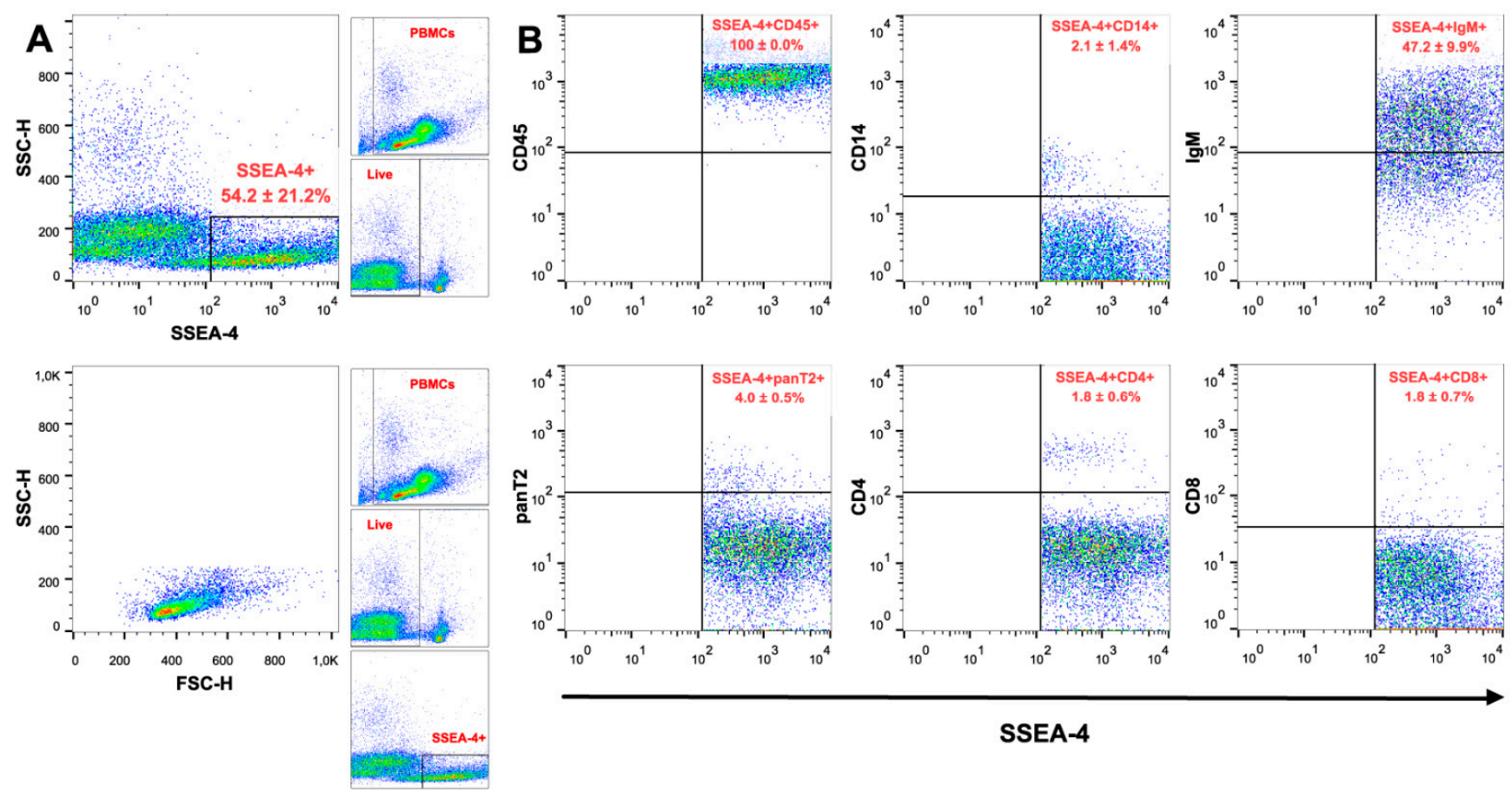

SSEA-4

Figure 1. Flow-cytometric strategy for the phenotyping of SSEA-4 ${ }^{+}$cells presented within peripheral blood mononuclear cells (PBMCs). Firstly, live PBMCs were gated and then number of SSEA-4 ${ }^{+}$cells was measured (A: upper dotplot). Backgating of SSEA $-4^{+}$cells revealed their location mainly within the lymphocyte region (A: lower dotplot). Phenotype of SSEA-4 gated cells were evaluated in terms of the expression of several leukocyte markers as follows: CD45, CD14, IgM, panT2, CD4 and CD8 (B). The data from four experiments are expressed as the means \pm SD.
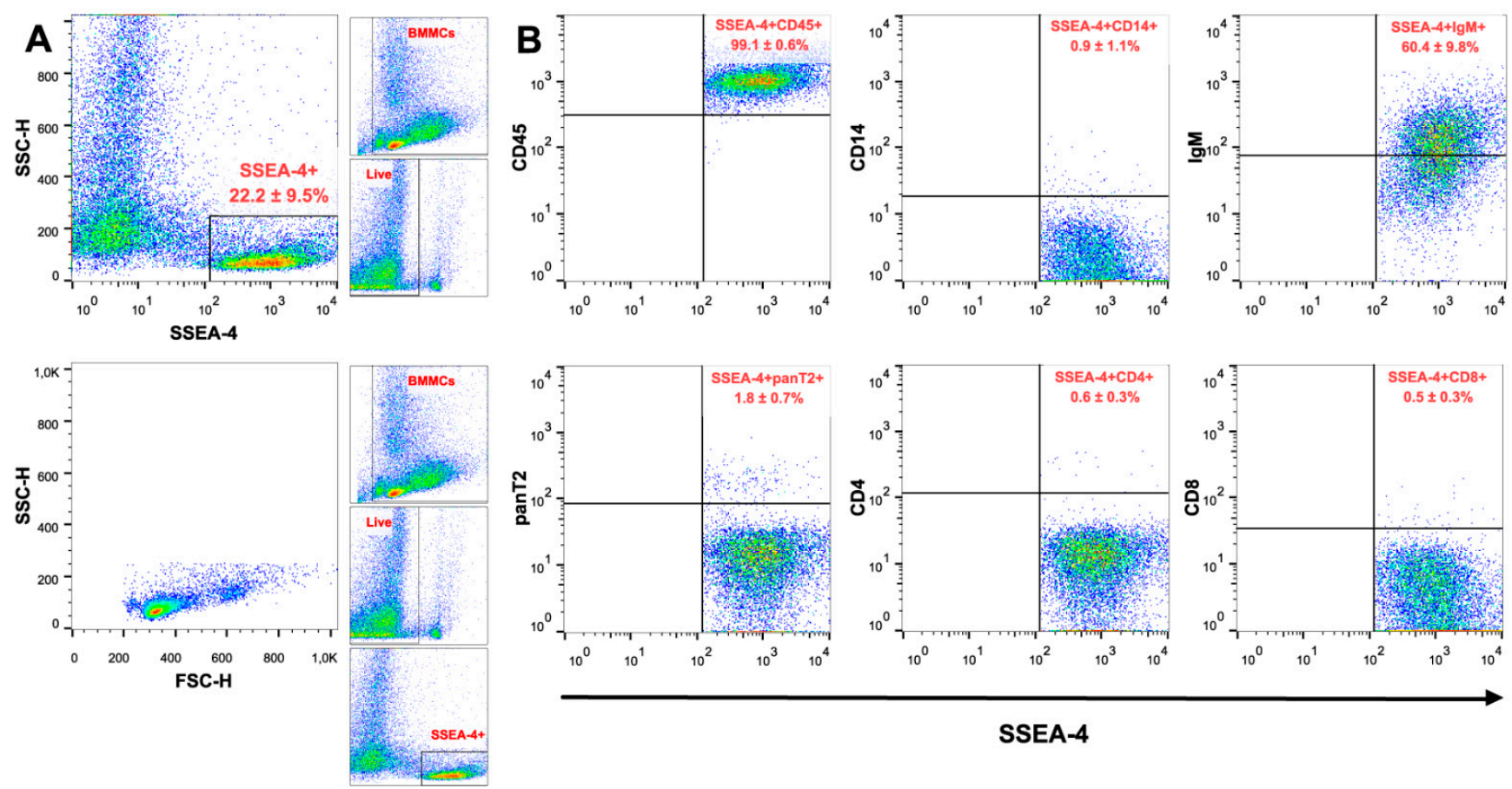

SSEA-4

Figure 2. Flow-cytometric strategy for the phenotyping of SSEA-4 ${ }^{+}$cells presented within bone marrow mononuclear cells (BMMCs). Firstly, live BMMCs were gated and then the number of SSEA- $4^{+}$cells was measured (A: upper dotplot). Backgating of SSEA $-4^{+}$cells revealed their location mainly within the lymphocyte region (A: lower dotplot). Phenotype of SSEA-4 gated cells were evaluated in terms of the expression of several leukocyte markers as follows: CD45, CD14, IgM, panT2, CD4 and CD8 (B). The data from five experiments are expressed as the means \pm SD. 
Table 1. Purity and efficiency of MACS enrichment of SSEA- $4^{+}$cells.

\begin{tabular}{|c|c|c|c|c|c|}
\hline Sample & Con $\left(\right.$ SSEA- $\left.4^{+}\right)$ & $\operatorname{Neg}\left(\right.$ SSEA-4$\left.^{+}\right)$ & Pos $\left(\right.$SSEA-4 $\left.4^{+}\right)$ & Pos $\left(\mathrm{SSEA}-4^{+} \mathrm{CD}^{2} 5^{+}\right)$ & Pos $\left(\mathrm{SSEA}-4^{+} \mathrm{LCR}^{+}\right)$ \\
\hline PBMCs & $58.6 \pm 16.0$ & $8.4 \pm 2.4^{* *}$ & $90.1 \pm 4.4^{*}$ & $89.5 \pm 3.4$ & $88.6 \pm 3.9$ \\
\hline BMMCs & $26.4 \pm 9.1$ & $5.2 \pm 1.5^{*}$ & $84.8 \pm 1.8^{* *}$ & $82.5 \pm 3.4$ & $80.7 \pm 3.0$ \\
\hline
\end{tabular}

The data are expressed as the mean \pm SD; PBMCs-peripheral blood mononuclear cells, BMMCs-bone marrow mononuclear cells, Con-fresh (control) unsorted sample, Neg-negative fraction, Pos-positive fraction, LCR-labeling check reagent. The data from four experiments are expressed as the means $\pm \mathrm{SD}$; ${ }^{*}$-difference is statistically significant at $p<0.05$; ${ }^{* *}$-difference is statistically significant at $p<0.01$ in comparison to control samples.

\subsection{Real-Time (qPCR) Analysis of SSEA-4 Sorted Cells}

Relative mRNA expression of selected markers such as SSEA-4, CD45 (leukocytes), CD14 (monocytes), CD79 $\alpha$ and IgM (B-cell markers), CD3g, CD4, CD8a and CD8b (Tcell markers), $\mathrm{MHCI}$ and $\mathrm{MHCII}$ in control (unsorted) samples and both sorted fractions (negative and positive) was analyzed using the qPCR method. In both cell types, PBMCs and BMMCs, expression of SSEA-4 significantly increased in the positive fractions in comparison to control samples (Figures 3 and 4). Furthermore, the positive fractions highly expressed CD45, whereas CD14 expression was significantly decreased when compared to control samples. Both cell types exhibited highly increased expression of IgM and CD79 $\alpha$ (B-cell markers) after sorting in positive fractions, though not significant in BMMCs for CD79 $\alpha$. On the other hand, a significant increase in the MHCII expression was observed in the positive fractions of BMMCs, while no significant change was noticed in PBMCs. The relative expressions of other evaluated markers (CD3g, CD4, CD8a, CD8b and MHCI) were not significantly changed after MACS sorting (Figures 3 and 4). However, higher standard deviations were observed for the T-cell markers in negative fractions of PBMCs and in positive fractions of BMMCs, which was probably caused by the higher variability of the analyzed data.

SSEA-4

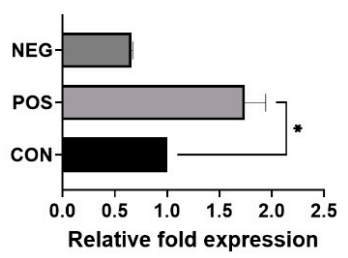

$\lg M$

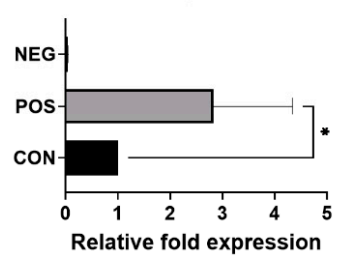

CD8b

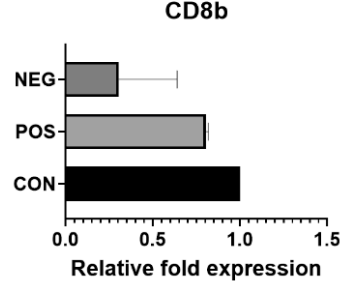

CD45

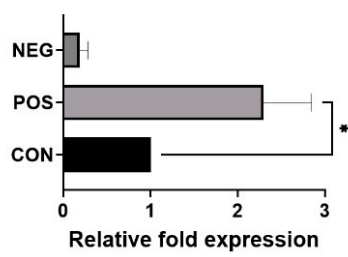

CD3g

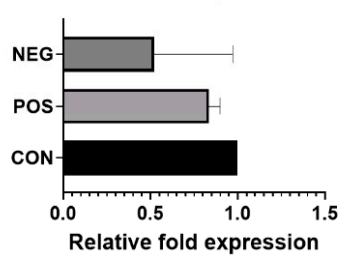

$\mathrm{MHCl}$

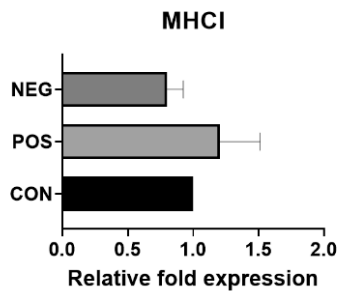

CD14

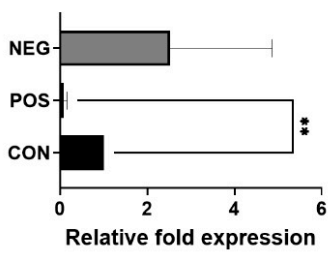

CD4
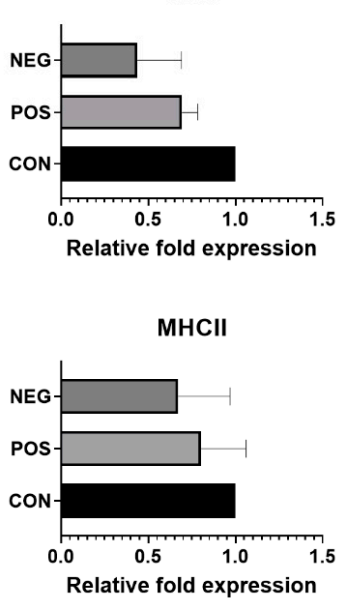

CD79a

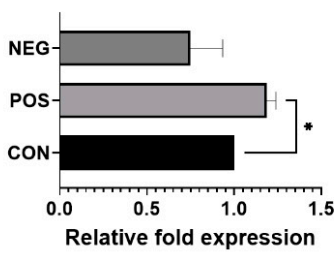

CD8a

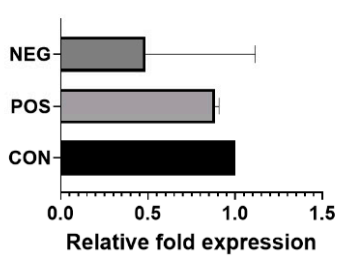

Figure 3. Relative expression of selected markers compared among control (unsorted) samples and sorted fractions (negative and positive) of PBMCs. Con—fresh (control) unsorted sample, Neg-negative fraction, Pos-positive fraction. The data from four experiments are expressed as the means $\pm \mathrm{SD} ;{ }^{*}$ - difference is statistically significant at $p<0.05$; ${ }^{* *}$-difference is statistically significant at $p<0.01$. 
SSEA-4

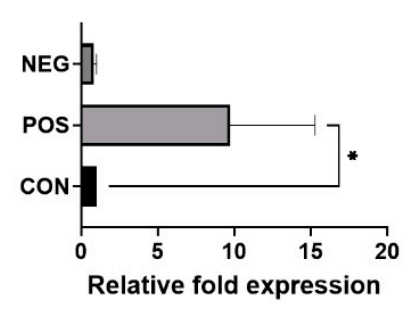

$\lg M$

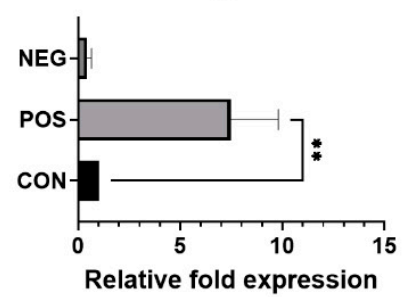

CD8b

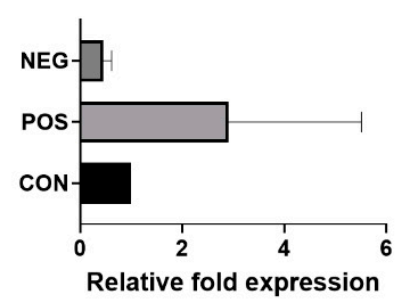

CD45

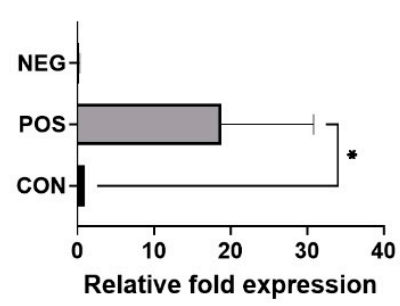

CD3g

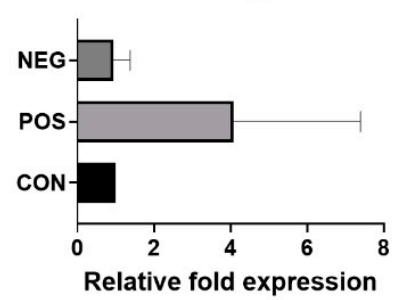

MHCI

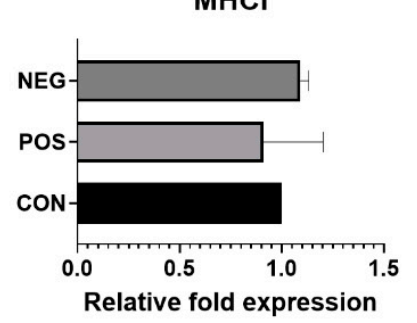

CD14

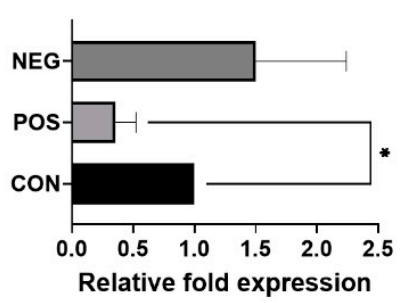

CD4

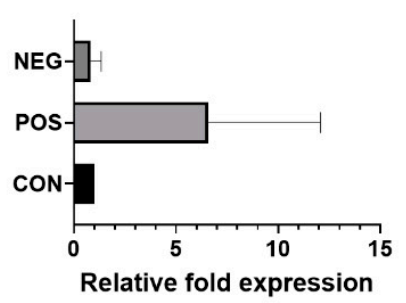

MHCII

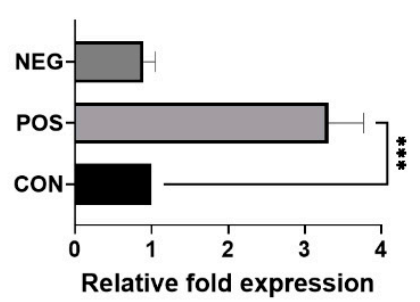

CD79a

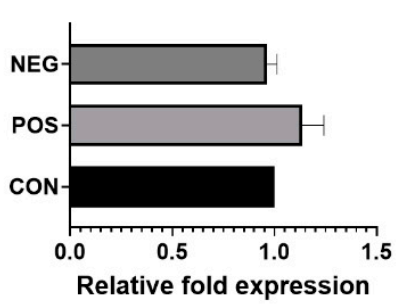

CD8a

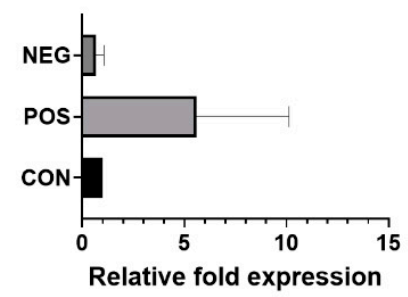

Figure 4. Relative expression of selected markers compared among control (unsorted) samples and sorted fractions (negative and positive) of BMMCs. Con—fresh (control) unsorted sample, Neg-negative fraction, Pos—positive fraction. The data from five experiments are expressed as the means $\pm \mathrm{SD} ;{ }^{*}$ - difference is statistically significant at $p<0.05$; ${ }^{* *}$-difference is statistically significant at $p<0.01 ; * *$ - difference is statistically significant at $p<0.001$.

\section{Discussion}

SSEA-3 and SSEA-4 are well-known as specific embryonic antigens. Both of them are epitopes located on the related glycosphingolipids (GSLs) which are marked as GL-5 and GL-7 [1]. The structure of GSLs comprises a carbohydrate moiety or chain which is linked to ceramide [22-24]. It has been demonstrated that SSEA-3 antibody (clone MC631) recognizes GL-5 as well as an internal antigen on GL-7. On the other hand, SSEA-4 antibody (clone MC813-70) recognizes only the terminal sialic acid on GL-7 [1].

In this study, we used antibody clone MC813-70 to identify and isolate SSEA- $4^{+}$cells presented in rabbit peripheral blood and bone marrow. Interestingly, almost a double number of SSEA $-4^{+}$cells were detected in PBMCs $(>50 \%)$ in comparison to BMMCs ( $>20 \%$; Figures 1 and 2, and Table 1). The decreased number of these cells in BMMC samples may be due to the fact that bone marrow is composed of the heterogenous mixture of hematopoietic stem and mature cells as well as of a stromal (nonhematopoietic) cell population [6]. Moreover, according to flow-cytometric analysis, BMMC samples were still contaminated with granulocytes (Figure 2A) while PBMCs samples contained mainly lymphocytes and monocytes with low granulocyte presence (Figure 1A). Furthermore, a similar proportion of SSEA- $4^{+}$cells (about $20 \%$ ) found here in rabbit BMMC samples was also observed in our previous study [21] or in human bone marrow samples when analyzed with the same antibody clone [25]. On the contrary, only $4 \%$ of human and $2 \%$ of mouse bone marrow cells were positive for SSEA-4 in another study [6]. This discrepancy might be due to the different SSEA-4 antibody used in that study. 
According to above mentioned studies, it is obvious that SSEA-4 expression is not restricted to the MSC population presented within BMMCs. This is supported by the weak SSEA-4 expression in rabbit MSCs [19] and by the failure to facilitate and accelerate the rabbit MSC culture via seeding of SSEA-4 enriched cells [21]. Moreover, the absolute majority of rabbit SSEA-4 ${ }^{+}$PBMCs and also BMMCs co-expressed CD45 (pan-leukocyte marker) as demonstrated by flow cytometry (Figures 1 and 2). An increased CD45 expression in both rabbit SSEA $-4^{+}$sorted fractions was also proved using qPCR analyses (Figures 3 and 4). SSEA $-4^{+}$CD $45^{+}$subpopulation has been even noticed in mouse bone marrow samples and, furthermore, about $50 \%$ of human SSEA $-4^{+}$enriched bone marrow cells also co-expressed CD45 [6].

Since the SSEA $-4^{+}$cells in rabbit blood and bone marrow seems to be of hematopoietic origin according to the CD45 expression and localization within the lymphogate (Figures 1 and 2), we used flow cytometry for the phenotyping of fresh unsorted (control) samples. Beside the expression of CD45, the SSEA $-4^{+}$cells from both sources were negative for monocyte marker (CD14) and all T-cell markers (panT2, CD4 and CD8). However, SSEA-4 gated cells exhibited similarly high levels of IgM (marker of immature B cells) expression ( $~ 50 \%$ or $60 \%$ for PBMCs and BMMCs, respectively). In order to confirm the flow-cytometric results, SSEA- $4^{+}$cells were enriched via MACS with significant efficiency (>80\%) in both rabbit cell types (Table 1) and their phenotypic expression was analyzed using qPCR analyses (Figures 3 and 4). The relative SSEA-4 and CD45 expression of SSEA $-4^{+}$sorted cells increased significantly $(p<0.05)$, while CD14 expression decreased ( $p<0.01$ and $p<0.05$, respectively) in comparison to fresh unsorted (control) samples. On the other hand, all T-cell markers (CD3g, CD4, CD8a and CD8b) did not significantly change, whereas an increased expression of B-cell markers (CD79 $\alpha$ and IgM) was observed in SSEA- $4^{+}$PBMCs $\left(p<0.05\right.$ for both markers) and SSEA $-4^{+}$BMMCs $(p<0.01$ for $\operatorname{IgM}$ only). Although no change in the relative expression of MHCI was observed, the expression of MHCII significantly $(p<0.001)$ increased in SSEA-4 $4^{+}$BMMCs, but not in PBMCs. Moreover, MHCII positivity has been reported to identify the B-cell lineage, as all CD79 $\alpha^{+}$ cells in the rabbit bone marrow are $\mathrm{MHCII}^{+}$and vice versa [26]. Thus, both phenotyping methods, flow cytometry and qPCR, demonstrated that cells expressing SSEA-4 epitope are of hematopoietic origin and the majority of them possess the phenotype of B lymphocytes. Moreover, the hematopoietic origin of SSEA-4 sorted cells was verified by microscopic assessment of SSEA-4 and CD45 co-expression (Appendix A). In addition, significantly increased expression of hematopoietic stem cell marker CD133 was observed in the positive fractions compared to the control samples. On the other hand, the expression of mesenchymal stem cell marker CD271 was not observed in the sorted cells (Appendix B). Thus, the mesenchymal origin of these cells is less probable than their hematopoietic origin. This hypothesis can be supported by the fact that the majority of round-shaped SSEA-4 sorted bone marrow cells were non-adherent as reported in our previous study [21]. Only a small proportion of SSEA-4 sorted cells adhered to the plastic and established a culture of rabbit mesenchymal stem cells (MSCs), although not more efficiently than the unsorted samples. Furthermore, rabbit bone marrow-derived MSCs demonstrated a weak expression of SSEA-4 marker, as noticed in another study [19].

Beside embryos and embryonic stem cells [1-4], SSEA-4 expression has been already observed in adult stem and progenitor cells [5-8,20] or even more also in differentiated cells [1,14-16]. In addition, SSEA-4 has been recently identified as a potential marker of tumor cells with migration capacity [27]. However, clinically healthy young rabbits that were randomly selected for the experiments were used in this study. Therefore, the probability that all rabbits chosen for this study were affected by some type of cancer is very low. On the other hand, we can hypothesize that SSEA-4 expression in this study could be associated with the presence of B cell progenitors (proB and preB cells) that are crucial for B lymphopoiesis in the bone marrow $[26,28]$ and/or with the differentiated B lymphocytes themselves. However, although B lymphopoiesis occurs in mouse or human bone marrow throughout life [26], it occurs early in rabbit development and only for a short time [29,30]. 
It has been noticed that levels of proB and preB cells rapidly decreased in young rabbits by 4 weeks after birth. Moreover, by 16 weeks of age, proB and preB cells were undetectable and B lymphocytes constituted all B-lineage cells in rabbit bone marrow. Thus, it seems that $99 \%$ of rabbit B lymphopoiesis is arrested and only few B lymphocytes are produced after 16 weeks of age in rabbits [26]. Altogether, this is an evidence that rabbit B lymphocytes must be long-lived and/or self-renewing [26,31,32]. SSEA-4 expression noticed in this study is therefore probably not associated with the presence of progenitor cells since rabbits at the age of 3-5 months used here should contain only few B cell progenitors. On the other hand, expression of SSEA-4 might be related to the self-renewing ability of rabbit B lymphocytes, though this hypothesis has to be proved by further experiments. Until then, the role of SSEA-4 antigen presented on rabbit lymphocytes and its function remains unknown.

\section{Materials and Methods}

\subsection{Animals}

Young (3-5 months old) and clinically healthy rabbits $(n=5)$ of New Zealand White (NZW) line reared as described previously [33] were used in this study. The treatment of the animals was approved by the Ministry of Agriculture and Rural Development of the Slovak Republic no. SK U 18016 in accordance with the ethical guidelines presented in Slovak Animal Protection Regulation (RD 377/12), which conforms to the Code of Ethics of the EU Directive 2010/63/EU for animal experiments.

\subsection{Experimental Design}

All experiments were designed to identify the SSEA $-4^{+}$cell population within the mononuclear cells of rabbit peripheral blood (PBMCs) and bone marrow (BMMCs) using flow cytometry. Then, SSEA $-4^{+}$cells were isolated from both sources by magnetic-activated cell sorting (MACS) and characterized using Real-Time PCR (qPCR) in order to confirm the phenotype observed by flow cytometry.

\subsection{Flow Cytometry}

Rabbit PBMCs and BMMCs were isolated as described previously [19,34]. Briefly, peripheral blood $(\mathrm{PB})$ and bone marrow $(\mathrm{BM})$ were isolated from humanely sacrificed animals. PB was collected to tubes with anticoagulant and diluted with sterile PBS. BM was flushed from femurs immediately after removal of femoral bone heads using sterile PBS. The cells suspension was filtrated in order to remove cell clumps and bone fragments. Mononuclear cells from both prepared suspensions, $\mathrm{PB}$ and BM, were isolated using Biocoll solution on the basis of density gradient centrifugation. Isolated PBMCs and BMMCs were stained with the mouse monoclonal antibodies against the following rabbit antigens: CD4, CD8, CD14, CD45, IgM (B-cell marker), pan T2 (T-cell marker) and SSEA-4. The staining procedure was performed as described in our previous study [33]. The list of primary antibodies used in this study is shown in Table 2. The proper isotype controls and secondary antibodies were used for the purified primary antibodies: mouse IgG3-PE isotype control (12-4742), rat anti-mouse IgG2a-FITC (clone m2a-15F8) and rat anti-mouse IgG1-APC (clone M1-14D12; all from eBioscience, Wien, Austria). To exclude the dead cells from the analysis, 7-AAD (Thermo Fisher Scientific, Waltham, MA, USA) staining was applied. At least 50,000 cells were analyzed per each sample by FACSCalibur flow cytometer (BD Biosciences, San Jose, CA, USA). Obtained data were analyzed using FlowJo ${ }^{\text {TM }}$ Software v10.7 (BD Biosciences, San Jose, CA, USA). 
Table 2. List of primary antibodies used for flow cytometry.

\begin{tabular}{ccccc}
\hline Marker & Host/Isotype & Clone & Conjugate & Company \\
\hline CD4 & mouse IgG1 & RTH1A & purified $^{1}$ & WSU \\
CD8 & mouse IgG2a & ISC27A & purified $^{1}$ & WSU \\
CD14 & mouse IgG2a & TÜK4 & FITC & Dako Cytomation \\
CD45 & mouse IgG1 & L12/201 & purified $^{1}$ & Bio-Rad \\
CD45 & mouse IgG2a & ISC18A & purified $^{1}$ & WSU \\
IgM & mouse IgG1 & NRBM & purified $^{1}$ & Bio-Rad \\
pan T2 & mouse IgG1 & RTH21A & purified $^{1}$ & WSU \\
SSEA-4 & mouse IgG3 & MC-813-70 & PE & eBioscience \\
\hline
\end{tabular}

${ }^{1}$ Cells stained with the purified antibodies were subsequently incubated with proper secondary antibodies.

\subsection{Magnetic-Activated Cell Sorting of SSEA-4 ${ }^{+}$Cells}

Both rabbit PBMCs and BMMCs were sorted as demonstrated previously [21]. Briefly, cells $\left(2 \times 10^{7}\right)$ from each sample were incubated with PE-conjugated SSEA-4 antibody (clone MC-813-70; eBioscience, Wien, Austria) according to the producer's manual. After the washing step, samples were immediately incubated with Anti-PE MicroBeads (Miltenyi Biotec, Bergisch Gladbach, Germany) and sorted by AutoMACS Pro Separator (Miltenyi Biotec, Bergisch Gladbach, Germany) using "POSSEL_S" sorting strategy (positive selection) according to the producer's manual. After sorting, cell aliquots from both fractions, positive (SSEA- $4^{+}$) and negative (SSEA- $4^{-}$), were co-stained with CD45 antibody (Table 2) and proper FITC-conjugated secondary as well as with APC-conjugated labeling check reagent (LCR; Miltenyi Biotec, Bergisch Gladbach, Germany) which specifically binds to magnetic beads. Fresh (control) unsorted cells were also stained with SSEA-4 and CD45 antibody, and also with LCR in order to determine initial SSEA $-4^{+} \mathrm{CD} 45^{+}$population and to exclude the unspecific binding of LCR. The subsequent flow-cytometric analysis performed as mentioned above revealed the sorting purity and efficiency as the proportion of SSEA- 4 single positive (SSEA- $4^{+}$) cells and SSEA-4/LCR double positive (SSEA- $4^{+} \mathrm{LCR}^{+}$) cells, respectively.

\subsection{Real-Time PCR ( $q P C R)$}

Total RNA isolation and cDNA synthesis from fresh (control) samples and both sorted fractions (SSEA $-4^{+}$and SSEA- $4^{-}$) was performed as described previously [35]. The rabbit gene-specific primers used in this study (Table 3) have been already published or designed de novo using the Primer-BLAST at NCBI's website [36]. A slightly modified qPCR reaction was performed as described previously [35]: initial denaturation and activation of hot-start DNA polymerase at $95^{\circ} \mathrm{C}$ for $7 \mathrm{~min}$ followed by 40 cycles of denaturation at $95^{\circ} \mathrm{C}$ for $10 \mathrm{~s}$, annealing at $60{ }^{\circ} \mathrm{C}$ for $10 \mathrm{~s}$ and extension at $72{ }^{\circ} \mathrm{C}$ for $10 \mathrm{~s}$ in Rotor-Gene 6000 (Corbett Research, Sydney, Australia). A relative quantification of gene expression to housekeeping gene beta 2-microglobulin (B2M) was calculated using the threshold (CT) values and PCR reaction efficiencies according to Pfaffl [37].

\subsection{Statistical Analysis}

Data obtained from analyses were evaluated using GraphPad Prism version 9.0.1 for Windows (GraphPad Software, San Diego, CA, USA) with one-way ANOVA (Dunnett's test). Results are expressed as the mean $\pm \mathrm{SD}$. $p$-values at $p<0.05$ were considered as statistically significant. 
Table 3. Gene-specific primers and size of PCR products.

\begin{tabular}{|c|c|c|c|c|c|}
\hline Gene & Product Size (bp) & Forward Primer & Reverse Primer & Primer Efficiency & Reference \\
\hline ST3GAL2 (SSEA-4) & 126 & 5'-CTGGGAGAATAACCGGTACG-3' & 5'-GCTCAGTTGCCTCGGTAGAC-3' & 2.04 & [19] \\
\hline CD 45 & 262 & $5^{\prime}$-TACTCTGCCTCCCGTTG-3' & $5^{\prime}$-GCTGAGTGTCTGCGTGTC-3' & 2.02 & [19] \\
\hline $\mathrm{CD} 14$ & 128 & $5^{\prime}$-TCTCTGTCCCCACAAGTTCC-3' & 5'-GGCTGAGGTCTAGGTGATGG-3' & 1.92 & NM_001082195.2 ${ }^{1}$ \\
\hline IGHM (IgM) & 127 & 5'-GCCTGTACTTCACCCACAGC-3' & 5'-GACTTGTCCACGGTCCTCTC-3' & 2.04 & MN651044.1 ${ }^{1}$ \\
\hline CD3g & 104 & 5'-TCATTGCAGGACAAGATGGA-3' & 5'-GTCATCTTCCCGATCCTTGA-3' & 2.01 & XM_002722697.3 ${ }^{1}$ \\
\hline CD4 & 163 & $5^{\prime}$-CAGTCCTGGGTCAGCTTCTC-3' & 5'-TATCAAGGGTCAGGCTCAGG-3' & 2.06 & NM_001082313.2 ${ }^{1}$ \\
\hline CD8a & 133 & 5'-GTGGACTCTCCGCTCAACTC-3' & 5'-GGCTGAAGTGTAGGCTGAGG-3' & 2.07 & XM_017340260.1 1 \\
\hline MHCI & 97 & 5'-AGTGGGAATTGTTGCTGGAG-3' & 5'-TCCTTTCCCATCTGAGCTGT-3' & 1.91 & [38] \\
\hline MHCII & 142 & $5^{\prime}$-CTGTGAGCACAGGAAGGTGA-3' & $5^{\prime}$-GGGTGGCTAGATGTCTGGAA-3' & 2.00 & [38] \\
\hline B2M & 118 & 5'-ATTCACGCCCAATGATAAGG-3' & 5'-ATCCTCAGACCTCCATGCTG-3' & 2.02 & [19] \\
\hline
\end{tabular}

${ }^{1}$ NCBI Reference Sequence. 


\section{Conclusions}

In this study, a significant number of cells expressing SSEA-4 antigen were discovered within the rabbit peripheral blood and bone marrow. Although SSEA-4 is considered to be mainly an antigen of embryonic and adult stem and progenitor cells, it could be found also in some specific types of differentiated cells as reported here. According to results from cell phenotyping, SSEA-4 positive cells are of hematopoietic origin (as co-expressed CD45) and possess B-cell phenotype (IgM, CD79 $\alpha$ and MHCII). As far as we know, this is the first study that demonstrated SSEA-4 expression in differentiated rabbit cells. However, the role of this marker in rabbit lymphocytes remain unknown, and further analyses such as RNA sequencing and/or CFU assays are required in order to discover its real function.

Author Contributions: J.V.: conceptualization, methodology, formal analysis, investigation, resources, data curation, writing-original draft preparation, writing-review and editing, supervision, project administration, funding acquisition. A.B.: investigation, writing-review and editing. M.B.: methodology, formal analysis, investigation, data curation. P.C.: writing-review and editing, supervision, project administration, funding acquisition. All authors have read and agreed to the published version of the manuscript.

Funding: This research was funded by the Slovak Research and Development Agency, grant number APVV-14-0348, APVV-17-0124 and APVV-18-0146 and by the Scientific Grant Agency of the Ministry of Education, Science, Research and Sport of the Slovak Republic and Slovak Academy of Sciences, grant number VEGA 1/0160/18.

Institutional Review Board Statement: The study was conducted according to the guidelines of the Declaration of Helsinki, and approved by the Ministry of Agriculture and Rural Development of the Slovak Republic no. SK U 18016 (31.11.2016) in accordance with the ethical guidelines presented in the Slovak Animal Protection Regulation (RD 377/12).

Informed Consent Statement: Not applicable.

Data Availability Statement: The data presented in this study are available in the article.

Acknowledgments: The authors would like to thank to Ján Pecho and Igor Matušica for their technical assistance.

Conflicts of Interest: The authors declare no conflict of interest.

\section{Appendix A}

Confocal Microscopy

To confirm the expression of SSEA- 4 and CD45 markers by SSEA- $4^{+}$sorted cells, a microscopic assessment under the laser scanning confocal microscope LSM 700 (Carl Zeiss Slovakia, Bratislava, Slovakia) was performed. Briefly, positively sorted (SSEA$4^{+}$) PBMCs and BMMCs, which were co-stained with SSEA-4 and CD45 antibodies as mentioned in Material and Methods (Section 4.4), were fixed using IC Fixation Buffer (Thermo Fisher Scientific, Waltham, MA, USA) for 20 min and washed. An aliquot from each fixed sample was mixed with the VECTASHIELD antifade mounting medium with DAPI (Vector Laboratories, Burlingame, CA, USA), dropped on the microscope slide and mounted with coverslip. Microscopic evaluation confirmed co-expression of SSEA-4 and CD45 markers in the positively sorted samples of PBMCs as well as BMMCs (Figure A1). 

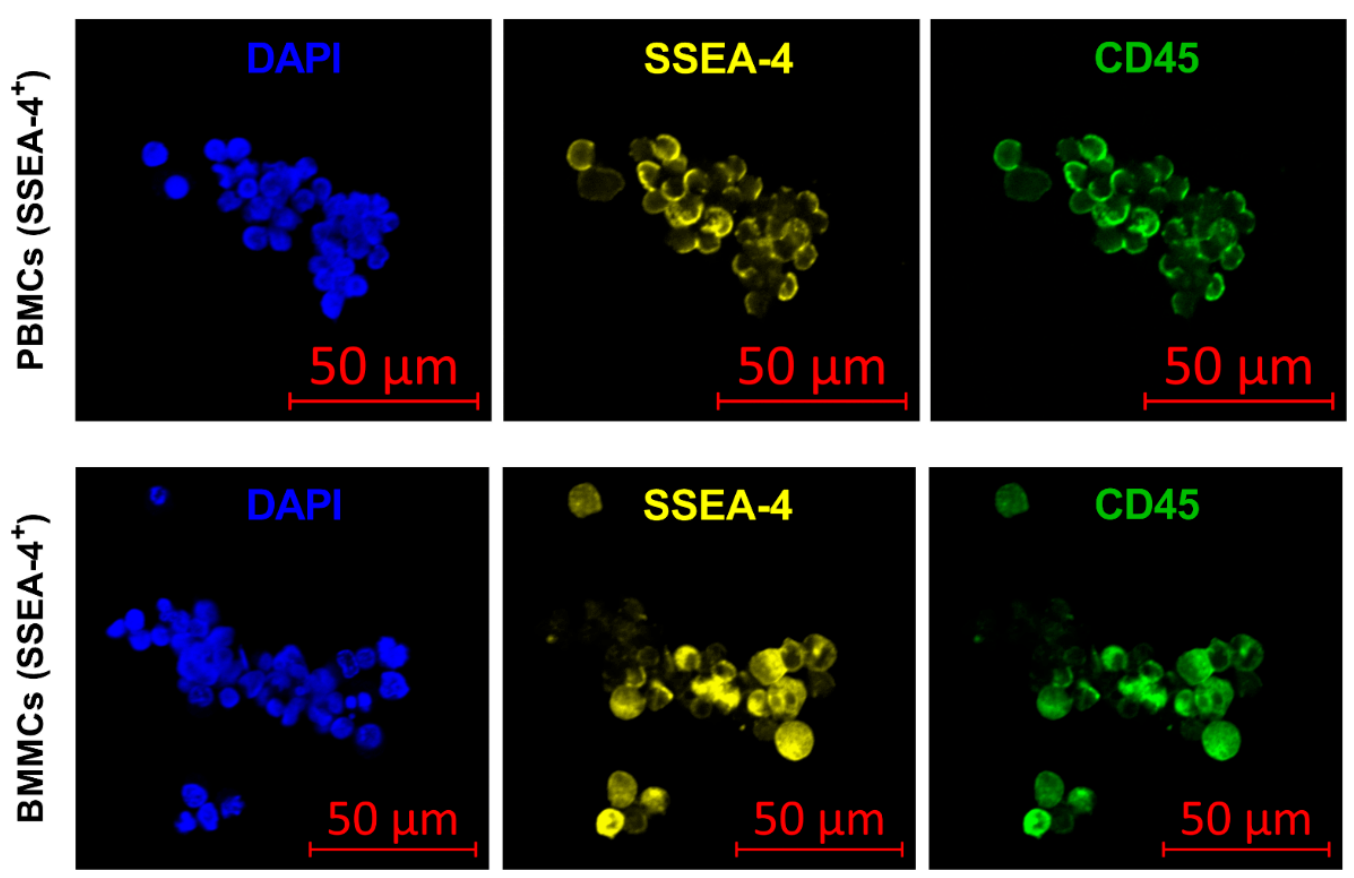

Figure A1. Immunofluorescent images of SSEA-4 positively sorted PBMCs and BMMCs. Blue—total nucleated cells stained with DAPI, yellow—cells positive for SSEA-4 and green—cells positive for CD45 (Scale bars $=50 \mu \mathrm{m}$ ).

\section{Appendix B}

In order to evaluate the origin and potential differentiation capacity of SSEA-4 sorted cells, a qPCR analysis was performed in order to assess the possible expression of typical hematopoietic stem cell marker (CD133) and mesenchymal stem cell marker (CD271). Briefly, samples for qPCR and the reaction itself were performed as mentioned in Material and Methods (Section 4.5). Rabbit gene-specific primers were used according to the previous studies for CD133 [39] and for CD271 [40]. SSEA-4 ${ }^{+}$fractions from both PBMC and BMMC samples significantly expressed CD133 in comparison to the control samples (Figure A2). On the other hand, no expression of CD271 was observed in the studied samples, although the specificity of used primers was confirmed by obtaining the specific PCR product for CD271 (117 bp) in rabbit DNA samples and in the sample of rabbit adipose-derived mesenchymal stem cells (Figure A2). 

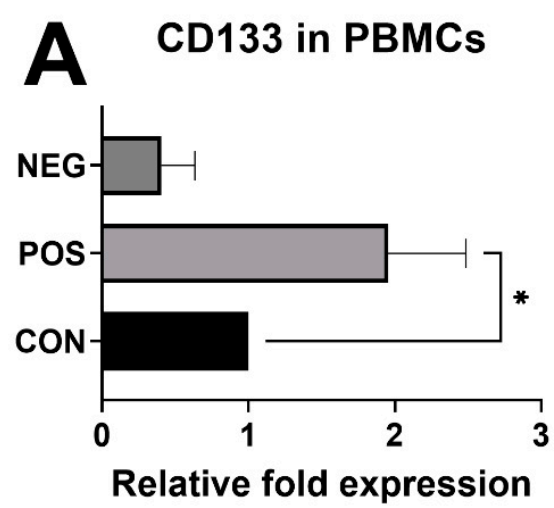

CD133 in BMMCs

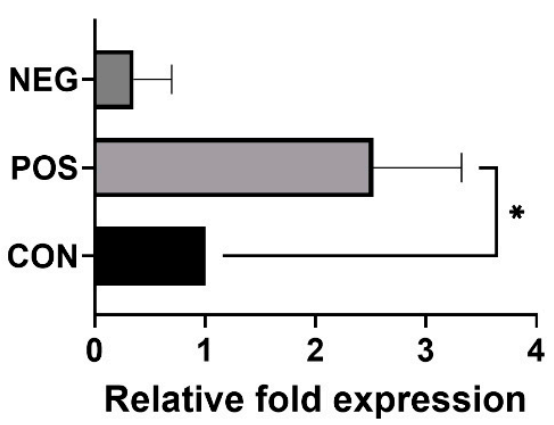

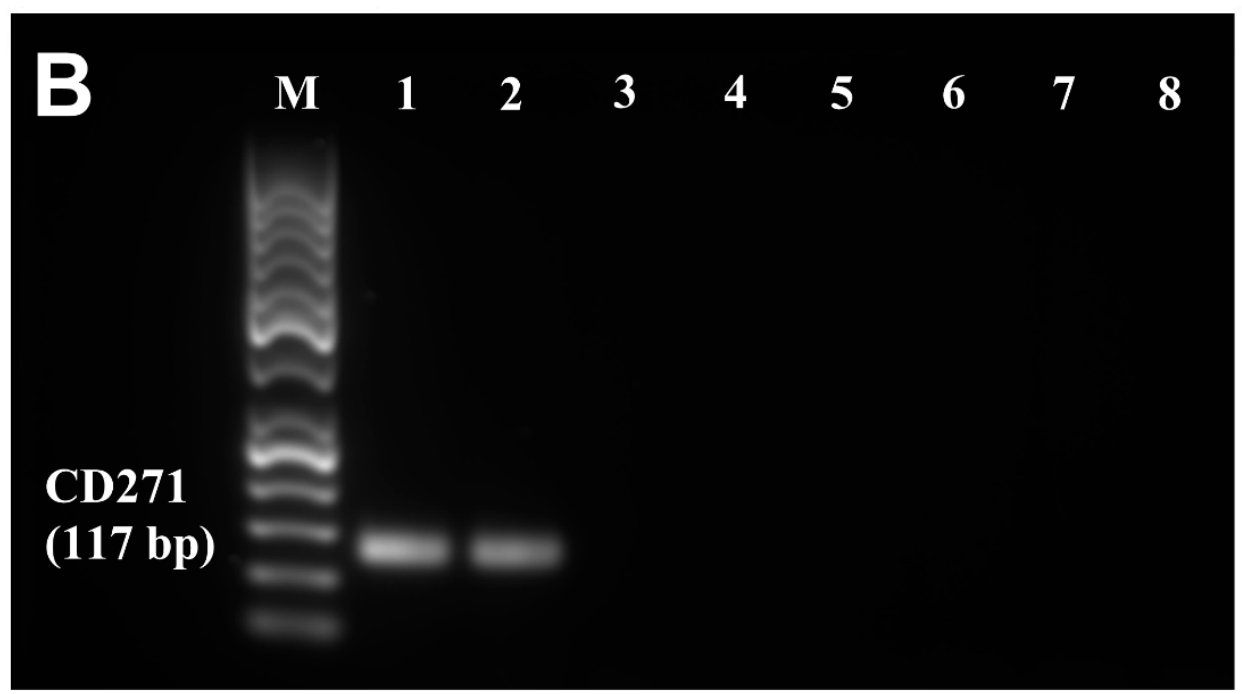

Figure A2. Expression of selected stem cell markers (CD133 and CD271) compared among control (unsorted) samples and sorted fractions (negative and positive) of PBMCs and BMMCs. Con-fresh (control) unsorted sample, Neg-negative fraction, Pos-positive fraction. (A) The data from four experiments are expressed as the means $\pm \mathrm{SD} ;{ }^{*}$ - difference is statistically significant at $p<0.05$. (B) Lane M-50 bp DNA ladder (Thermo Fisher Scientific, USA); lane 1-rabbit DNA; lane 2-rabbit adipose-derived mesenchymal stem cells; lane 3-PBMCs control (unsorted) sample; lane 4-positive PBMCs fraction; lane 5-negative PBMCs fraction; lane 6-BMMCs control (unsorted) sample; lane 7-positive BMMCs fraction; lane 8-negative BMMCs fraction.

\section{References}

1. Kannagi, R.; Cochran, N.A.; Ishigami, F.; Hakomori, S.; Andrews, P.W.; Knowles, B.B.; Solter, D. Stage-specific embryonic antigens (SSEA-3 and -4) are epitopes of a unique globo-Series ganglioside isolated from human teratocarcinoma cells. EMBO J. 1983, 2, 2355-2361. [CrossRef] [PubMed]

2. Muramatsu, T.; Muramatsu, H. Carbohydrate antigens expressed on stem cells and early embryonic cells. Glycoconj. J. 2004, 21, 41-45. [CrossRef] [PubMed]

3. Wright, A.J.; Andrews, P.W. Surface marker antigens in the characterization of human embryonic stem cells. Stem Cell Res. 2009, 3, 3-11. [CrossRef]

4. Suila, H.; Pitkanen, V.; Hirvonen, T.; Heiskanen, A.; Anderson, H.; Laitinen, A.; Natunen, S.; Miller-Podraza, H.; Satomaa, T.; Natunen, J.; et al. Are globoseries glycosphingolipids SSEA-3 and-4 markers for stem cells derived from human umbilical cord blood? J. Mol. Cell Biol. 2011, 3, 99-107. [CrossRef]

5. $\quad$ Bhartiya, D.; Shaikh, A.; Nagvenkar, P.; Kasiviswanathan, S.; Pethe, P.; Pawani, H.; Mohanty, S.; Rao, S.G.A.; Zaveri, K.; Hinduja, I. Very Small Embryonic-Like Stem Cells with Maximum Regenerative Potential Get Discarded During Cord Blood Banking and Bone Marrow Processing for Autologous Stem Cell Therapy. Stem Cells Dev. 2012, 21, 1-6. [CrossRef]

6. Gang, E.J.; Bosnakovski, D.; Figueiredo, C.A.; Visser, J.W.; Perlingeiro, R.C.R. SSEA-4 identifies mesenchymal stem cells from bone marrow. Blood 2007, 109, 1743-1751. [CrossRef] [PubMed] 
7. Sandstedt, J.; Jonsson, M.; Vukusic, K.; Dellgren, G.; Lindahl, A.; Jeppsson, A.; Asp, J. SSEA-4+CD34-Cells in the Adult Human Heart Show the Molecular Characteristics of a Novel Cardiomyocyte Progenitor Population. Cells Tissues Organs 2014, 199, 103-116. [CrossRef]

8. Afrikanova, I.; Kayali, A.; Lopez, A.; Hayek, A. Is Stage-Specific Embryonic Antigen 4 a Marker for Human Ductal Stem/Progenitor Cells? Biores. Open Access 2012, 1, 184-191. [CrossRef]

9. He, H.P.; Nagamura-Inoue, T.; Tsunoda, H.; Yuzawa, M.; Yamamoto, Y.; Yorozu, P.; Agata, H.; Tojo, A. Stage-Specific Embryonic Antigen 4 in Wharton's Jelly-Derived Mesenchymal Stem Cells Is Not a Marker for Proliferation and Multipotency. Tissue Eng. Part A 2014, 20, 1314-1324. [CrossRef]

10. Kuroda, Y.; Kitada, M.; Wakao, S.; Nishikawa, K.; Tanimura, Y.; Makinoshima, H.; Goda, M.; Akashi, H.; Inutsuka, A.; Niwa, A.; et al. Unique multipotent cells in adult human mesenchymal cell populations. Proc. Natl. Acad. Sci. USA 2010, 107, 8639-8643. [CrossRef]

11. Wakao, S.; Kitada, M.; Kuroda, Y.; Dezawa, M. Isolation of Adult Human Pluripotent Stem Cells from Mesenchymal Cell Populations and Their Application to Liver Damages. In Liver Stem Cells: Methods and Protocols; Methods in Molecular Biology; Ochiya, T., Ed.; Humana Press Inc.: Totowa, NJ, USA, 2012; Volume 826, pp. 89-102.

12. Brimble, S.N.; Sherrer, E.S.; Uhl, E.W.; Wang, E.; Kelly, S.; Merrill, A.H.; Robins, A.J.; Schulz, T.C. The cell surface glycosphingolipids SSEA-3 and SSEA-4 are not essential for human ESC pluripotency. Stem Cells 2007, 25, 54-62. [CrossRef]

13. Yamashita, T.; Wada, R.; Sasaki, T.; Deng, C.X.; Bierfreund, U.; Sandhoff, K.; Proia, R.L. A vital role for glycosphingolipid synthesis during development and differentiation. Proc. Natl. Acad. Sci. USA 1999, 96, 9142-9147. [CrossRef]

14. Dodd, J.; Solter, D.; Jessell, T.M. Monoclonal antibodies against carbohydrate differentiation antigens identify subsets of primary sensory neurones. Nature 1984, 311, 469-472. [CrossRef]

15. Truong, T.T.; Huynh, K.; Nakatsu, M.N.; Deng, S.X. SSEA4 Is a Potential Negative Marker for the Enrichment of Human Corneal Epithelial Stem/Progenitor Cells. Investig. Ophthalmol. Vis. Sci. 2011, 52, 6315-6320. [CrossRef]

16. Mueller, T.; Eildermann, K.; Dhir, R.; Schlatt, S.; Behr, R. Glycan stem-cell markers are specifically expressed by spermatogonia in the adult non-human primate testis. Hum. Reprod. 2008, 23, 2292-2298. [CrossRef] [PubMed]

17. Rozemuller, H.; Prins, H.J.; Naaijkens, B.; Staal, J.; Buhring, H.J.; Martens, A.C. Prospective Isolation of Mesenchymal Stem Cells from Multiple Mammalian Species Using Cross-Reacting Anti-Human Monoclonal Antibodies. Stem Cells Dev. 2010, 19, 1911-1921. [CrossRef] [PubMed]

18. Ding, Z.; Huang, H. Mesenchymal stem cells in rabbit meniscus and bone marrow exhibit a similar feature but a heterogeneous multi-differentiation potential: Superiority of meniscus as a cell source for meniscus repair. BMC Musculoskelet. Disord. 2015, 16, 14. [CrossRef] [PubMed]

19. Vasicek, J.; Kovac, M.; Balazi, A.; Kulikova, B.; Tomkova, M.; Olexikova, L.; Curlej, J.; Bauer, M.; Schnabl, S.; Hilgarth, M.; et al. Combined approach for characterization and quality assessment of rabbit bone marrow-derived mesenchymal stem cells intended for gene banking. New Biotechnol. 2020, 54, 1-12. [CrossRef]

20. Barraud, P.; Stott, S.; Mollgard, K.; Parmar, M.; Bjorklund, A. In vitro characterization of a human neural progenitor cell coexpressing SSEA4 and CD133. J. Neurosci. Res. 2007, 85, 250-259. [CrossRef]

21. Vasicek, J.; Balazi, A.; Chrenek, P. The efficiency of immunomagnetic sorting of rabbit bone marrow cells for the establishment of mesenchymal stem cell culture. J. Microbiol. Biotechnol. Food Sci. 2018, 8, 890-892. [CrossRef]

22. Futerman, A.H.; Hannun, Y.A. The complex life of simple sphingolipids. EMBO Rep. 2004, 5, 777-782. [CrossRef]

23. Merrill, A.H. De novo sphingolipid biosynthesis: A necessary, but dangerous, pathway. J. Biol. Chem. 2002, $277,25843-25846$. [CrossRef]

24. Shayman, J.A. Sphingolipids. Kidney Int. 2000, 58, 11-26. [CrossRef] [PubMed]

25. Sobiesiak, M.; Sivasubramaniyan, K.; Hermann, C.; Tan, C.; Orgel, M.; Treml, S.; Cerabona, F.; de Zwart, P.; Ochs, U.; Muller, C.A.; et al. The Mesenchymal Stem Cell Antigen MSCA-1 is Identical to Tissue Non-specific Alkaline Phosphatase. Stem Cells Dev. 2010, 19, 669-677. [CrossRef]

26. Jasper, P.J.; Zhai, S.K.; Kalis, S.L.; Kingzette, M.; Knight, K.L. B lymphocyte development in rabbit: Progenitor B cells and waning of B lymphopoiesis. J. Immunol. 2003, 171, 6372-6380. [CrossRef] [PubMed]

27. Sivasubramaniyan, K.; Harichandan, A.; Schilbach, K.; Mack, A.F.; Bedke, J.; Stenzl, A.; Kanz, L.; Niederfellner, G.; Buhring, H.J. Expression of stage-specific embryonic antigen-4 (SSEA-4) defines spontaneous loss of epithelial phenotype in human solid tumor cells. Glycobiology 2015, 25, 902-917. [CrossRef]

28. Shahaf, G.; Zisman-Rozen, S.; Benhamou, D.; Melamed, D.; Mehr, R. B Cell Development in the Bone Marrow Is Regulated by Homeostatic Feedback Exerted by Mature B Cells. Front. Immunol. 2016, 7, 13. [CrossRef]

29. McElroy, P.J.; Willcox, N.; Catty, D. Early precursors of B lymphocytes I. Rabbit/mouse species differences in the physical properties and surface phenotype of pre-B cells, and in the maturation sequence of early B cells. Eur. J. Immunol. 1981, 11, 76-85. [CrossRef]

30. Crane, M.A.; Kingzette, M.; Knight, K.L. Evidence for limited B-lymphopoiesis in adult rabbits. J. Exp. Med. 1996, 183, $2119-2127$. [CrossRef]

31. Mage, R.G.; Lanning, D.; Knight, K.L. B cell and antibody repertoire development in rabbits: The requirement of gut-associated lymphoid tissues. Dev. Comp. Immunol. 2006, 30, 137-153. [CrossRef] [PubMed] 
32. Kalis, S.L.; Zhai, S.K.; Yam, P.C.; Witte, P.L.; Knight, K.L. Suppression of B lymphopoiesis at a lymphoid progenitor stage in adult rabbits. Int. Immunol. 2007, 19, 801-811. [CrossRef]

33. Kovac, M.; Vasicek, J.; Kulikova, B.; Bauer, M.; Curlej, J.; Balazi, A.; Chrenek, P. Different RNA and protein expression of surface markers in rabbit amniotic fluid-derived mesenchymal stem cells. Biotechnol. Prog. 2017, 33, 1601-1613. [CrossRef]

34. Vasicek, J.; Shehata, M.; Schnabl, S.; Hilgarth, M.; Hubmann, R.; Jager, U.; Bauer, M.; Chrenek, P. Critical Assessment of the Efficiency of CD34 and CD133 Antibodies for Enrichment of Rabbit Hematopoietic Stem Cells. Biotechnol. Prog. 2018, 34, 1278-1289. [CrossRef] [PubMed]

35. Kulikova, B.; Kovac, M.; Bauer, M.; Tomkova, M.; Olexikova, L.; Vasicek, J.; Balazi, A.; Makarevich, A.V.; Chrenek, P. Survivability of rabbit amniotic fluid-derived mesenchymal stem cells post slow-freezing or vitrification. Acta Histochem. 2019, 121, 491-499. [CrossRef]

36. Ye, J.; Coulouris, G.; Zaretskaya, I.; Cutcutache, I.; Rozen, S.; Madden, T.L. Primer-BLAST: A tool to design target-specific primers for polymerase chain reaction. BMC Bioinform. 2012, 13, 11. [CrossRef] [PubMed]

37. Pfaffl, M.W. A new mathematical model for relative quantification in real-time RT-PCR. Nucleic Acids Res. 2001, 29, 6. [CrossRef] [PubMed]

38. Vašíček, J.; Baláži, A.; Bauer, M.; Svoradová, A.; Tirpáková, M.; Ondruška, L'; Parkányi, V.; Makarevich, A.V.; Chrenek, P. Enrichment of Rabbit Primitive Hematopoietic Cells via MACS Depletion of CD45+ Bone Marrow Cells. Magnetochemistry 2021, 7, 11. [CrossRef]

39. Vasicek, J.; Balazi, A.; Bauer, M.; Svoradova, A.; Tirpakova, M.; Tomka, M.; Chrenek, P. Molecular Profiling and Gene Banking of Rabbit EPCs Derived from Two Biological Sources. Genes 2021, 12, 366. [CrossRef] [PubMed]

40. Sanchez-Rodriguez, A.; Arias-Alvarez, M.; Rebollar, P.G.; Bautista, J.M.; Lorenzo, P.L.; Garcia-Garcia, R.M. Gene expression and immunolocalization of low-affinity neurotrophin receptor (p75) in rabbit male reproductive tract during sexual maturation. Reprod. Domest. Anim. 2018, 53, 62-65. [CrossRef] 